\title{
Alteration in Shoulder Kinematics and Associated Muscle Activity in People With Idiopathic Scoliosis
}

\author{
Jiu-jenq Lin, PT, PhD, * Wei-Hsiu Chen, PT, MS,† Po-Quang Chen, MD, and Jau-Yih Tsauo, PT, PhD*
}

Study Design. A cross-sectional control-matched study in adolescents with idiopathic scoliosis (IS).

Objective. To evaluate whether subjects with IS had shoulder dysfunction and associated impairment in shoulder kinematics and muscular activation.

Summary of Background Data. Evidence indicates that shoulder kinematics are related to shoulder pain and dysfunction. Despite the degree of morbidity associated with altered shoulder kinematics likely to occur in subjects with IS, no report has been published to address this hypothesis.

Methods. In this investigation, shoulder kinematics (scapular tipping, scapular upward rotation, and scapulohumeral rhythm) and associated muscular activities [upper trapezius (UT), lower trapezius (LT), serrantus anterior (SA), and middle deltoid (MD)] were evaluated with a 3-dimensional electromagnetic tracking device and electromyography during arm elevations in 13 female subjects with IS and 13 age-gender-dominant hand-matched controls. Additionally, self-reported Flexilevel Scale of Shoulder Function (FLEX-SF) was evaluated between the 2 groups.

Results. Subjects with IS demonstrated lower FLEX-SF scores than the controls $(P=0.01)$. For the convex side, more anterior tilt of the scapula in resting position was identified $(P=0.006)$. For the concave side, more scapular upward rotation in resting position was identified $(P=$ 0.01). For the EMG amplitude, higher LT contraction activity on the convex side and lower LT and SA contraction activity on the concave side were established $(P=0.007$ and less than 0.01 , respectively). A moderate positive association was found between scapular posterior tipping during movement and FLEX-SF functional score $(R=$ 0.51). A moderate negative association was found between LT muscular activity and FLEX-SF functional score $(R=-0.54)$.

Conclusion. Given the progressive nature of IS and kinematic linkage among the thoracic spine, scapula, and

From the "School and Graduate Institute of Physical Therapy, College of Medicine, National Taiwan University; †Department of Physical Therapy, Taipei Medical University Hospital; and $\ddagger$ Department of Orthopedic Surgery, National Taiwan University and Hospital, Taipei, Taiwan.

Acknowledgment date: February 18, 2009. Revision date: August 3, 2009. Acceptance date: August 4, 2009.

The manuscript submitted does not contain information about medical device(s)/drug(s).

Institutional funds were received in support of this work. No benefits in any form have been or will be received from a commercial party related directly or indirectly to the subject of this manuscript.

This study has been approved by the Institutional Review Board from University Hospital. Each subject signed an informed consent form approved by an Institutional Review Board.

Address correspondence and reprint requests to Jau-Yih Tsauo, School and Graduate Institute of Physical Therapy, College of Medicine, National Taiwan University, 3F, No. 17, Xu-Zhou Road, Taipei 100, Taiwan; E-mail: jytsauo@ntu.edu.tw arm, inadequate posterior tipping movement and high LT muscular activity on the convex side of IS are important to consider in rehabilitation programs for subjects with IS.

Key words: idiopathic scoliosis, shoulder, scapula, electromyography, motion analysis. Spine 2010;35: 1151-1157

Altered shoulder kinematics can be a source of pain and dysfunction for subjects with idiopathic scoliosis (IS). ${ }^{1-3}$ Change in shoulder kinematics has been associated with clinical symptoms, reduced functional capacity, and impaired quality of life. ${ }^{4,5}$ Postural abnormalities are often presented in subjects with IS and characterized as pelvic obliquity or tilt, shoulder tilt, and lateral shift of the upper thoracic vertebrae. ${ }^{1,2}$ Among these abnormalities, asymmetry of the scapula has been suggested as a predicator of overall spinal curvature in these patients. ${ }^{1}$ Thus, the degree of morbidity associated with altered shoulder kinematics is likely to be comparable with complex spinal curvature in subjects with IS.

Shoulder kinematics involves 3-dimensional movements of the humerus and the scapula, and change of shoulder kinematics is related to a variety of clinical conditions, ranging from minor impingement to shoulder dislocation. ${ }^{4,6,7}$ Although postural abnormalities, asymmetries in the frontal or transverse plane, are often noted in subjects with IS, the static, or limited 2-dimensional assessment may be unable to reveal information that is important in the clinical decision-making process. During arm elevation, the humerus rotates around the scapula at the glenohumeral joint, and the scapula moves into posterior tipping and upward rotation movements at the scapulothoracic joint. ${ }^{4,6,7}$ The result is a synchronized movement of the humerus and the scapula, described as the scapulohumeral rhythm. ${ }^{8}$ Kinematic changes related to scapulohumeral rhythm have been observed in subjects with a diagnosis of glenohumeral instability. ${ }^{9}$ Inadequate posterior tipping and upward rotation of the scapula during arm elevation have also been noted in subjects with shoulder impingement and shoulder dysfunction. ${ }^{4,5}$ Thus, we hypothesized that shoulder dysfunction and altered kinematics related to clinical symptoms would be present in subjects with IS.

Alterations in shoulder kinematics may be related to a particular dysfunctional pattern of muscular coordination. In fact, during arm elevation, the humerus moves about a movable scapula by action of the scapulothoracic muscles. ${ }^{10}$ These muscles stabilize the glenoid and locate the scapula dynamically for capable glenohumeral 


\section{Table 1. Subject Demographics}

\begin{tabular}{lcc}
\hline & $\begin{array}{c}\text { Scoliosis } \\
(\mathrm{N}=13)\end{array}$ & $\begin{array}{c}\text { Control } \\
(\mathrm{N}=13)\end{array}$ \\
\hline Age $(\mathrm{yr})$ & $16.0 \pm 3.8$ & $18.2 \pm 4.5$ \\
Dominant hand & Right: 13 & Right: 13 \\
Main thoracic* & Right: 13 & Right: 13 \\
Cobb anglet (degrees) & $34.0 \pm 7.3$ & - \\
FLEX-SF‡ & $35.5 \pm 4.6$ & 50 \\
\hline
\end{tabular}

*The main thoracic curve is the major curve, and the proximal thoracic and thoracolumbar/lumbar curves are minor curves.

tCobb's angle, a measurement used for evaluation of curves in scoliosis on an AP radiographic projection of the spine. Range from $31^{\circ}$ to $50^{\circ}$ was defined as moderate scoliosis [1].

\#Flexilevel scale of shoulder function (FLEX-SF): scores were recorded from 1 , with the most limited function, to 50 , without any limited function in the subject.

motion. Because subjects with IS are expected to have changed length-tension curves in scapulothoracic muscles, which may develop during adaptation to abnormal spinal curvature, ${ }^{11}$ altered kinematics and shoulder dysfunction can occur. Specifically, scapular functional stability depends on the proper activation of scapulothoracic muscles, particularly the order of muscular onset. ${ }^{12}$ Any small changes in this pattern of muscular dexterity can produce movement dysfunction at the glenohumeral joint, such as instability or impingement. ${ }^{13}$ Therefore, we also hypothesized that inadequate activation of scapulothoracic muscles would be present in subjects with IS.

The purposes of this research were 3-fold: (1) to identify whether subjects with IS had shoulder dysfunction or not; (2) to determine altered shoulder kinematics and associated activation of scapulothoracic muscles in subjects with IS as compared to controls; (3) to verify whether the degree of shoulder dysfunction is related to altered shoulder kinematics and/or activation of scapulothoracic muscles in subjects with IS.

\section{- Materials and Methods}

A cross-sectional research design was employed. Thirteen female subjects with idiopathic scoliosis and 13 age-genderdominant-hand-matched female subjects without scoliosis were recruited (Table 1). For a 2 -tailed test and a power of 0.80 , we estimated 13 subjects for a 10 FLEX-SF functional score difference between the 2 groups from our pilot study. Inclusion criteria were (1) age of 12 to 25 years old; (2) adolescent idiopathic scoliosis not previously treated; (3) major thoracic curve with a Cobb angle exceeding $21^{\circ}$ on an AP radiographic projection of the spine. Subjects who had concomitant cervical radiculopathy, evidence of bone spurs on radiographs, a history of surgery on the back or shoulders, traumatic injury, or pain during arm elevation were excluded. Each subject signed an informed consent form approved by an Institutional Review Board.

\section{Instrumentation}

The FASTRAK motion analysis system (Polhemus Inc., Colchester, VT) was used. This system includes sensors, a transmitter, motion capture units, a personal computer, and 6-D research software (Skill Technologies Inc., Phoenix, AZ).
The sensors for the system were attached to bony landmarks with adhesive tape. These surface sensor placements were the sternum, the flat superior bony surface of the scapular acromial process, and the distal humerus between the lateral and medial epicondyles. A fourth sensor attached to a stylus was used to digitize palpated anatomic coordinates (bony landmarks: sternal notch, xiphoid process, seventh cervical vertebra, 12 thoracic vertebrae, acromioclavicular joint, root of the spine of the scapula, inferior angle of the scapula, lateral epicondyle, and medial epicondyle; glenohumeral joint rotation center was operationalized by anterior humeral joint and posterior humeral joint). The absolute axes defined by the sensors were converted to anatomically defined axes derived from digitalized bony landmarks. Raw kinematic data were low-pass filtered at $6 \mathrm{~Hz}$.

The surface EMG assemblies include pairs of silver chloride circular (recording diameter of $10 \mathrm{~mm}$ ) surface electrodes (The Ludlow Company LP, Chocopee) with an interelectrode distance of $20 \mathrm{~mm}$, and a Grass AC/DC amplifier (Model 15A12, Astro-Med Inc., RI) with a gain of 1000 , a common mode rejection ratio of $86 \mathrm{~dB}$ at $60 \mathrm{~Hz}$, and a bandwidth $(-3 \mathrm{~dB})$ of 10 to $1000 \mathrm{~Hz}$. The sEMG data were collected at $2000 \mathrm{~Hz} /$ channel using a 16-bit analog to digital converter (Model MP150, Biopac systems Inc., CA). The impedance of each electrode was measured with respect to the reference electrode using an impedance meter (Model F-EZM5, Astro-Med Inc., RI). Each electrode was controlled to impedance of less than 10 k[Omega]. Full bandwidth sEMG data captured by the data acquisition software (AcqKnowledge, Biopac systems Inc., CA) was reduced using a root mean square (RMS) algorithm to produce sEMG envelopes with an effective sampling rate of 20 samples.

\section{Procedure}

After signing the informed consent form, the subjects were examined by a physical therapist to establish the clinical conditions of their shoulders, including range of motion and the Flexilevel scale of shoulder function (FLEX-SF) questionnaire (Scores were recorded from 1, with the most limited function, to 50, without any limited function in the subject). Then the patients changed into sports bras. The sensors for the motioncapturing system were attached to the bony landmarks. Surface electrodes were placed over the upper trapezius (UT) muscle (two thirds of the distance from the spinous process of the seventh cervical vertebra to the acromion process), the lower trapezius (LT) muscle (one fourth of the distance from the thoracic spine to the inferior angle of the scapula with the arm elevated in the sagittal plane), the lower serratus anterior (SA) muscle (over the muscle fibers anterior to the latissimus dorsi muscle with the arm elevated $90^{\circ}$ in the sagittal plane), and the middle deltoid (MD) muscle (halfway between the tip of the acromion and the deltoid tubercle). ${ }^{14}$ The skin was prepared by aggressive scrubbing with alcohol pads. A reference electrode was placed on the distal ulna of the other wrist. Verification of signal quality was investigated for each muscle by having the subject perform a resisted contraction in manual muscle test positions specific to each muscle of interest. ${ }^{15}$

Kinematics and EMG data were collected for 5 seconds in the resting seated posture with arms relaxed at the sides. Subjects were then asked to perform abduction with the scapular plane oriented $40^{\circ}$ anterior to the coronal plane. ${ }^{8}$ Three replicated movements were performed to the maximum motions. A tone signal was given when the subjects were to start the arm movement. An event-timer switch was used by the investiga- 
tor to mark the beginning of the arm movement. The event timer generated an electrical signal that was collected with the FASTRAK and EMG systems simultaneously. The high reliability of this approach has been described previously. ${ }^{7,16}$ All of the tests were made within the recommended space after calibration. ${ }^{17}$

\section{Data Reduction}

The original, full-bandwidth EMG data were reduced to an envelope of the activity using a root mean square (RMS) algorithm. Averages of activity during each analyzed event were computed from the RMS values. A vector was generated as RMS values from UT, LT, SA, and MD as response vector (RV). Magnitude was defined as the sum of RMS values from 4 muscles. Additionally, normalization of RV (formula) was conducted to represent muscle contraction pattern among 4 muscles during the task. Thus, similarity index (SI) was calculated as the inner product of normalization of RV and prototype RV, an average of the normalized magnitude number based on the collected data of every target trial of 13 healthy subjects. When the muscle contraction pattern was similar, SI value was close to 1 ; if the muscle contraction pattern was dissimilar, SI value was close to 0 . This method has been validated previously. ${ }^{18-20}$

To determine muscle timing, it is necessary to determine when a muscle is

$$
\mathrm{R}_{\text {norm }}=\frac{\left[\mathrm{R}_{1} \mathrm{R}_{2} \mathrm{R}_{3} \mathrm{R}_{4}\right]}{\sqrt{\sum_{i} \mathrm{R}_{i}^{2}}},
$$

where $\mathrm{R}_{1}=$ upper trapezius, $\mathrm{R}_{2}=$ lower trapezius, $\mathrm{R}_{3}=$ serratus anterior, and $\mathrm{R}_{4}=$ middle deltoid, considered "active." $\mathrm{A}$ muscle was considered "active" when the level of muscle was greater than 3 standard deviations above the baseline reference. The baseline reference was a 100-millisecond period before the onset of the movement signal in the resting seated posture. The onset of muscle activity was determined for each muscle.

The local coordinate system was developed from the digitized anatomic landmarks for the trunk and humerus and was used to describe clinically relevant motions of the shoulder. ${ }^{6,8,16,21}$ Humeral orientation relative to the thorax was described using an Euler angle sequence in which the first rotation represents the plane of elevation, the second rotation defines the amount of elevation, and the third rotation describes the amount of axial rotation. Scapular orientation relative to the thorax was described using an Euler angle sequence of rotation about $Z_{\mathrm{s}}$ (protraction/retraction), rotation about $\mathrm{Y}_{\mathrm{s}}$ (downward/upward rotation), and rotation about $\mathrm{X}_{\mathrm{s}}$ (posterior/anterior tipping). To quantitatively characterize shoulder and scapular kinematics, the scapulo-humeral rhythm and the scapular tilt at the humeral elevation of $120^{\circ}$ were used as dependent variables.

\section{Data Analysis}

The Shapiro-Wilk test confirmed that the data were normally distributed. To determine the difference between the 2 groups, independent $t$ tests were used to test EMG magnitude, similarity index, muscle activation time, scapulohumeral rhythm, scapular tilt, and FLEX-SF scores. A significant $\alpha$ level of 0.05 was used. In subjects with scoliosis, Pearson product-moment coefficients of correlations were also used to correlate scores of FLEX-SF and EMG/kinematics (EMG magnitude, similarity index, scapulohumeral rhythm, scapular tilt). In general, a correlation coefficient $(r)$ of 0 to 0.25 was considered to show little or no correlation, 0.25 to 0.50 to show a fair correlation, 0.5 to
Table 2. Means and Standard Deviations for Scapulohumeral Rhythm, Scapular Tilt

\begin{tabular}{lcc}
\hline & $\begin{array}{c}\text { Scoliosis Convex } \\
\text { Side (Right Side) }\end{array}$ & $\begin{array}{c}\text { Control } \\
\text { (Right Side) }\end{array}$ \\
\hline $\begin{array}{l}\text { Resting position } \\
\text { Scapular anterior tilt }\end{array}$ & $10.4 \pm 4.6^{*}$ & $2.4 \pm 5.4$ \\
$\begin{array}{l}\text { Scapular upward rotation } \\
\text { Abduction in the scapular plane } \\
\text { Scapular posterior tipping }\end{array}$ & $14.3 \pm 6.2$ & $12.4 \pm 3.9$ \\
Scapulohumeral rhythm & $12.1 \pm 6.0$ & $10.4 \pm 7.2$ \\
& $0.39 \pm 0.14$ & $0.41 \pm 0.19$ \\
& Scoliosis Concave & \\
& Side (Left Side) & (Left Side) \\
\hline $\begin{array}{l}\text { Resting position } \\
\text { Scapular posterior tilt }\end{array}$ & $3.9 \pm 6.9$ & $1.8 \pm 6.7$ \\
$\quad$ Scapular upward rotation & $6.2 \pm 6.4^{*}$ & $12.4 \pm 5.7$ \\
Abduction in the scapular plane & & \\
Scapular posterior tipping & $3.8 \pm 4.0$ & $5.6 \pm 6.8$ \\
Scapulohumeral rhythm & $0.23 \pm 0.16$ & $0.29 \pm 0.08$ \\
\hline * $P<0.05$. & & \\
\hline
\end{tabular}

0.75 to show a moderate to good correlation, and above 0.75 to show a good to excellent correlation. ${ }^{22}$

\section{- Results}

The descriptive statistics for SI, EMG magnitude, muscle activation time, scapulohumeral rhythm, and scapular tilt for both sides and both groups are summarized in Tables 2 and 3. Subjects with idiopathic scoliosis demonstrated significantly lower FLEX-SF scores compared with the control ( $P=0.01$, Table 1$)$. The shoulder on the convex side had dysfunction (a difference of 15) and supported our first hypothesis that subjects with idiopathic scoliosis would have shoulder dysfunction on the convex side of the spine.

A significant difference between shoulder kinematics was found. For the convex side, more anterior tilt of the scapula in resting position was identified $(P=0.006$, Table 2). There was no significant difference in scapular upward rotation in resting position, scapular posterior tipping, or scapulohumeral rhythm during movement between groups on the convex side. For the concave side, more scapular upward rotation in resting position was identified ( $P=0.01$, Table 2$)$. There was no significant difference in scapular tilt, scapular upward rotation, and scapulohumeral rhythm between groups on the concave side. Thus, the second hypothesis was partially supported.

A significant difference between EMG profiles was found. Less similarity was found on both the convex and concave sides. For the EMG amplitude, abnormal LT and SA muscular activities were established (higher LT contraction activity in convex side and lower LT and SA contraction activity in concave side, $P=0.007$ and less than 0.01 respectively, Table 3$)$. For the muscle activation time, delayed onset was identified on LT, SA, and MD on the convex side $(P<0.01$, Table 3$)$, and delayed onset was identified on UT, LT, and SA on the concave 
Table 3. Means and Standard Deviations for EMG Magnitude and Muscle Activation Time

\begin{tabular}{|c|c|c|c|c|c|c|c|c|}
\hline & \multicolumn{4}{|c|}{ Scoliosis Convex Side (Right Side) } & \multicolumn{4}{|c|}{ Control (Right Side) } \\
\hline \multirow[t]{2}{*}{ Similarity index } & \multicolumn{4}{|c|}{$0.86 \pm 0.07^{*}$} & \multicolumn{4}{|c|}{$0.92 \pm 0.03$} \\
\hline & UT & LT & SA & MD & UT & LT & SA & MD \\
\hline \multirow[t]{2}{*}{$\begin{array}{l}\text { EMG magnitude (\%) } \\
\text { Muscle activation time (ms) }\end{array}$} & $\begin{array}{l}30 \pm 11 \\
58 \pm 24\end{array}$ & $\begin{aligned} 29 & \pm 10^{*} \\
101 & \pm 60^{*}\end{aligned}$ & $\begin{array}{c}10 \pm 5 \\
117 \pm 35^{*}\end{array}$ & $\begin{array}{l}32 \pm 10 \\
56 \pm 27^{*}\end{array}$ & $\begin{array}{l}32 \pm 13 \\
46 \pm 13\end{array}$ & $\begin{array}{l}21 \pm 7 \\
60 \pm 23\end{array}$ & $\begin{array}{l}13 \pm 6 \\
86 \pm 21\end{array}$ & $\begin{array}{l}34 \pm 9 \\
40 \pm 9\end{array}$ \\
\hline & \multicolumn{4}{|c|}{ Scoliosis Concave Side (Left Side) } & \multicolumn{4}{|c|}{ Control (Left Side) } \\
\hline \multirow[t]{2}{*}{ Similarity index } & \multicolumn{4}{|c|}{$0.88 \pm 0.04^{*}$} & \multicolumn{4}{|c|}{$0.91 \pm 0.02$} \\
\hline & UT & LT & SA & MD & UT & LT & SA & MD \\
\hline $\begin{array}{l}\text { EMG magnitude } \\
\text { Muscle activation time (ms) }\end{array}$ & $\begin{array}{l}31 \pm 11 \\
71 \pm 30^{*}\end{array}$ & $\begin{array}{c}13 \pm 3^{*} \\
153 \pm 56^{*}\end{array}$ & $\begin{array}{c}15 \pm 6^{*} \\
148 \pm 56^{*}\end{array}$ & $\begin{array}{l}43 \pm 11 \\
64 \pm 18\end{array}$ & $\begin{array}{l}29 \pm 7 \\
48 \pm 21\end{array}$ & $\begin{array}{l}21 \pm 6 \\
78 \pm 38\end{array}$ & $\begin{array}{c}10 \pm 7 \\
105 \pm 38\end{array}$ & $\begin{array}{l}39 \pm 11 \\
52 \pm 30\end{array}$ \\
\hline
\end{tabular}

side $(P<0.03$, Table 3$)$. Accordingly, altered muscular activation partially supported the second hypothesis.

Associations were demonstrated in Figures 1 and 2. A moderate positive association was found between scapular posterior tipping during movement and FLEX-SF functional score $(R=0.51)$. A moderate negative association was found between LT muscular activity and FLEX-SF functional score $(R=-0.54)$. A fair positive association was identified between similarity index and FLEX-SF functional score $(R=0.37)$. A fair negative association was identified between SA muscular activity and FLEX-SF functional score $(R=-0.28)$. Small associations were found between scapulohumeral rhythm, UT muscular activity, MD muscular activity and FLEX-SF functional score $(R=-0.01,-0.03$, and 0.18 , respectively).

\section{Discussion}

Because the thoracic spine, scapula, and arm are links in the kinematic sequence of upper quadrant movements, any dysfunction is believed to be associated with others among the links. ${ }^{23-25}$ In our investigation, subjects with thoracic curve idiopathic scoliosis (IS) demonstrated moderate shoulder dysfunction (FLEX-SF: 35.5/50) on the convex side of the scoliosis curve. This shoulder dysfunction was also associated with scapula kinematics and muscular activities. For subjects with IS, deformities present at skeletal maturity will persist for life and may progress over time. In one study of 187 patients followed for more than 15 years, initial curvatures of $30^{\circ}$ to $39^{\circ}$ progressed $12^{\circ}$ further. ${ }^{26}$ Thus, our findings on subjects with IS (average curvature $=34^{\circ}$ ) for shoulder dysfunction and associated scapular anterior tilt/muscular contraction coordination impairments are important to consider in rehabilitation of subjects with IS.

The scapula resting position results showed different tipping positions in subjects with IS as compared with controls. Our results are in agreement with previous studies that demonstrated asymmetry of the scapula in a group predominantly consisting of thoracic IS. ${ }^{1,26}$ Previous authors, however, did not determine the impairment relative to control. In our study, the scapula on the convex side of curvature was in a more anteriorly tipped position, whereas the scapula on the concave side was in a tipping position similar to that of controls. A more anteriorly tipped position would leave the anterior acromion in nearer proximity to the rotator cuff tendons and increase the likely for impingement. Although posterior tipping of the scapula occurred during arm elevation to prevent impingement under the anterior acromial edge, inadequate posterior tipping movement would result in impingement of the rotator cuff tendons. The finding of a positive moderate association between posterior tipping movement and FLEX-SF scores supports the impingement phenomenon.

As the main movement of the scapula, upward rotation of the scapula has been most frequently addressed in clinical treatment approaches and research studies. ${ }^{4,8,10}$ Decreased upward rotation was believed to result in an inability to raise the lateral acromial edge of the scapula to obtain adequate clearance of the rotator cuff tendons during humeral elevation. In our study, an increased scapular upward rotation position was found on the concave side of the thoracic curvature. Subsequently, shoulder dysfunction did not occur on the concave side of the thoracic curvature. Although shoulder dysfunction was observed on the convex side of the thoracic curvature, we did not observe any difference in scapular upward rotation position and scapulohumeral rhythm during movement in a comparison of the convex side and the controls. Thus, shoulder dysfunction based on upward rotation of the scapula is not likely in subjects with IS.

Although the subjects with IS had altered muscular contraction strategies on both the concave and convex sides, increased muscular activities in the LT muscle on the convex side is important to consider in rehabilitation. Evidence suggests that raised concentrations of calmodulin, a calcium-binding protein, result in altered skeletal 

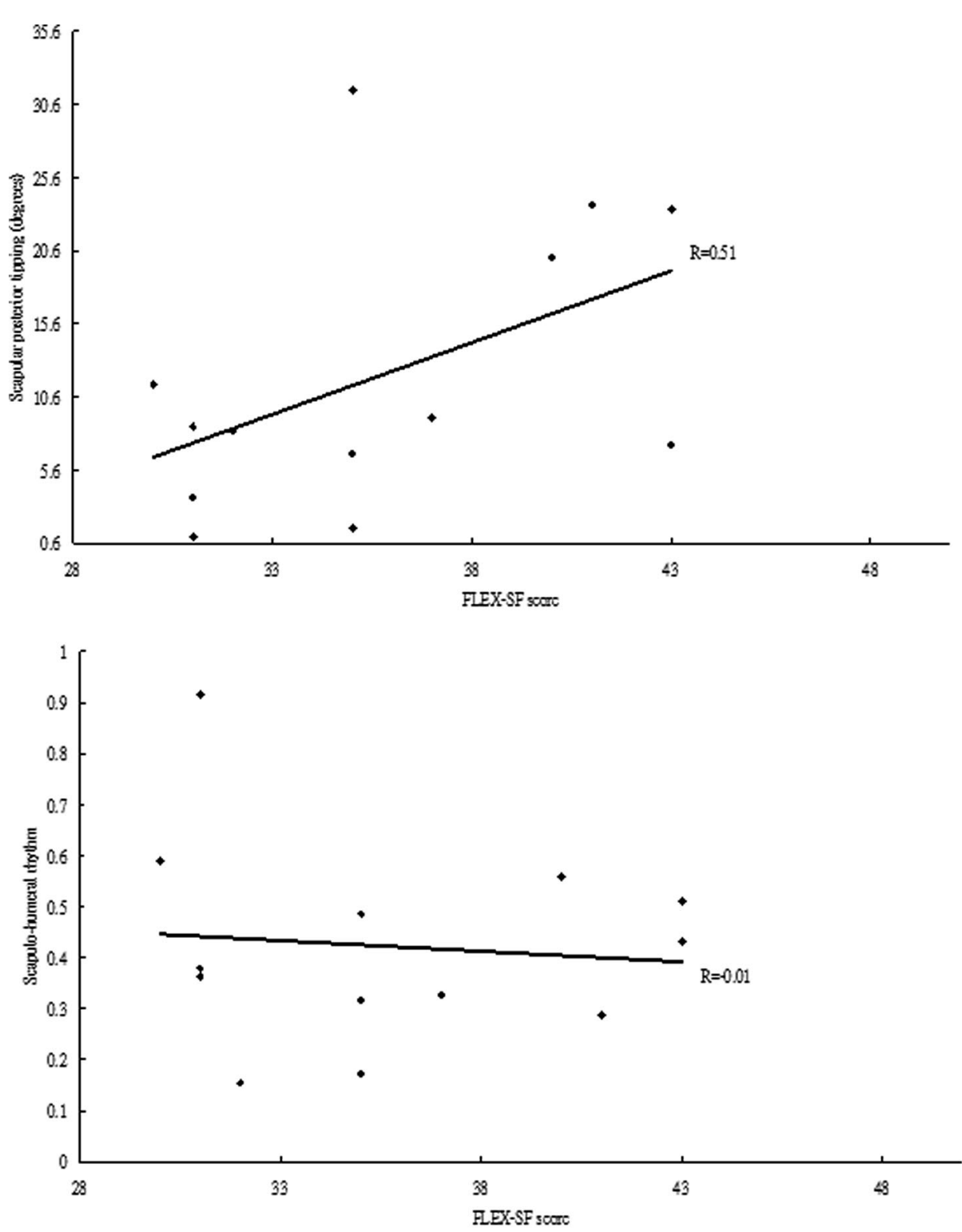

Figure 1. Scatter diagram showing relationship of shoulder kinematics/contraction similarity to flexilevel scale of shoulder function (FLEX-SF) in subjects with idiopathic scoliosis. The solid line represents the least-squares regression.

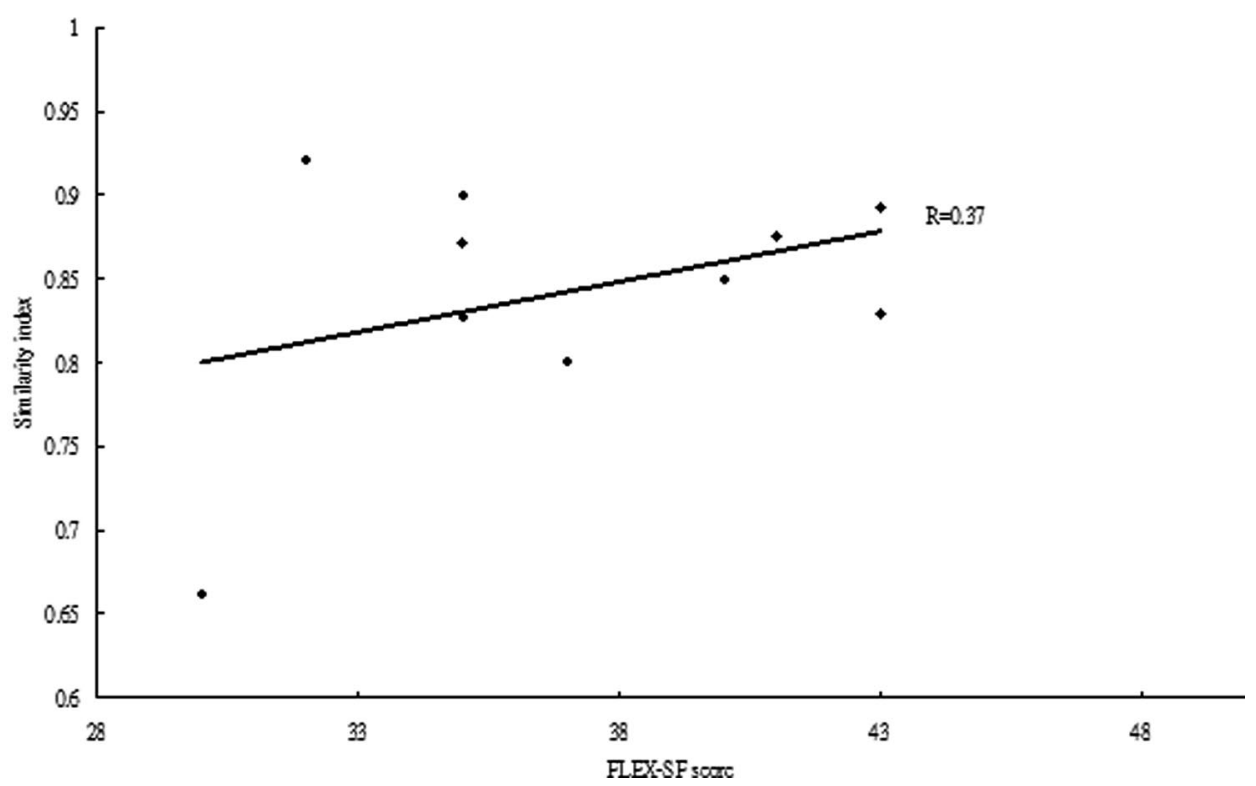



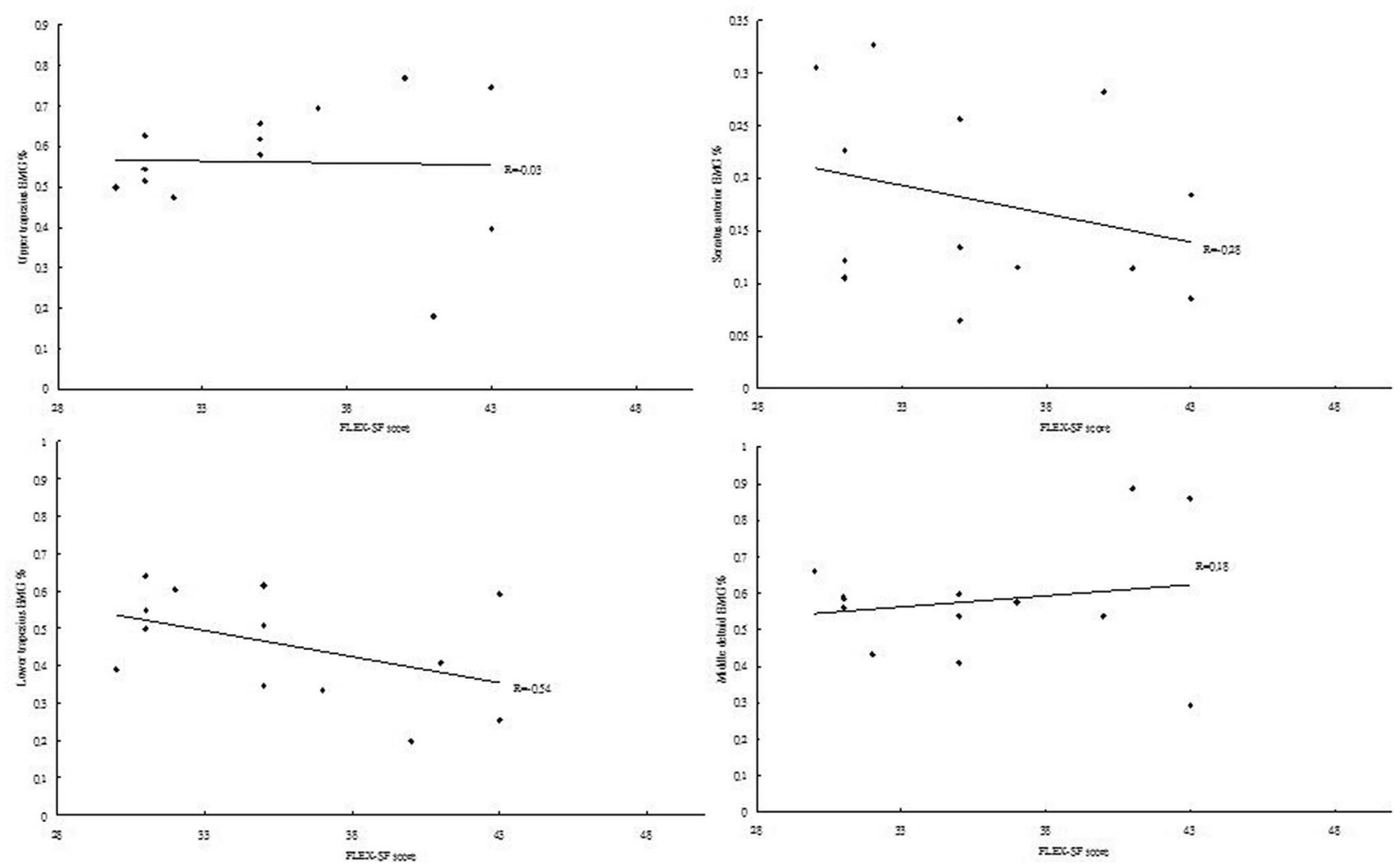

Figure 2. Scatter diagram showing relationship of muscle contraction activity to flexilevel scale of shoulder function (FLEX-SF) in subjects with idiopathic scoliosis. The solid line represents the least-squares regression.

muscle activity, imbalances in force production of scapular muscles, and subsequent progressive curvature in subjects with IS. ${ }^{27}$ In agreement with previous studies, delayed muscular onset and a lower similarity index were found on both sides in our study. The compensation strategies, however, are different on the 2 sides. The decreased LT and increased SA muscular activities are well balanced on the concave side because the kinematics, scapulohumeral rhythm, and shoulder functional status were not different from those in the controls. On the other hand, the increased LT, on the convex side, is believed to afford the muscular force to produce posterior tipping of the scapula and stabilize the scapular inferior angle against the thorax during humeral elevation. This phenomenon compensates for scapular anterior tilt in the resting posture on the convex side. The negative associations between LT muscular activities and functional ability scores from our data suggest that the increases in LT activation on the convex side were not able to adequately correct the anterior tilt position, resulting in a lack of posterior tipping and potential impingement and functional disability. Considering the hypothesized clinical importance of LT muscular activity as related to shoulder functional disability, the anterior tilt resting position and increased LT activity on the convex side in subjects with IS may be particularly relevant.

Limitations of the study should be noted. The skin motion artifact, which is associated with musculature and subcutaneous fat between skin and bone, is one lim- itation of a skin-based approach. Karduna et $a l^{6}$ indicated that data collected with the acromion method would be acceptable if humeral elevation stayed below $120^{\circ}$. In light of this recommendation, we analyzed the data in arm elevations of less than $120^{\circ}$. Potential alteration of the EMG signal because of muscle movement and cross talk should also be considered. To prevent such alteration, the electrode placements were chosen to minimize the cross talk from muscles. As deformities present at skeletal maturity may persist for life and may progress over time in these subjects, results may be different in studies of subjects with progressive scoliosis. Furthermore, the major thoracic curves with average Cobb angles of $34^{\circ}$ were relatively moderate, so subjects with more/less Cobb angle deformity might be expected to show more/less substantial shoulder functional disability as well as alterations in kinematics or muscle activity.

\section{- Key Points}

- Subjects with idiopathic scoliosis had shoulder dysfunction on the convex side of the spine.

- More anterior tilt of the scapula in resting position and positive moderate association between posterior tipping movement and FLEX-SF scores supports the impingement phenomenon on the convex side of the spine. 
- Increases in LT activation on the convex side were not able to adequately correct the anterior tilt position, resulting in a lack of posterior tipping and potential impingement and functional disability in subjects with IS.

- An inadequate posterior tipping movement and high LT muscular activity on the convex side of the curve are important to consider in rehabilitation programs for subjects with IS.

\section{References}

1. Raso VJ, Lou E, Hill DL, et al. Trunk distortion in adolescent idiopathic scoliosis. J Pediatr Orthop 1998;18:222-6.

2. Zabjek KF, Leroux MA, Coillard C, et al. Postural characteristics of adolescents with idiopathic scoliosis. J Pediatr Orthop 2008;28:218-24.

3. Masso PD, Gorton GE III. Quantifying changes in standing body segment alignment following spinal instrumentation and fusion in idiopathic scoliosis using an optoelectronic measurement system. Spine 2000;25:457-62.

4. Ludewig PM, Cook TM. Alterations in shoulder kinematics and associated muscle activity in people with symptoms of shoulder impingement. Phys Ther 2000;80:276-91.

5. Lin JJ, Hanten WP, Olson SL, et al. Shoulder-related dysfunction assessment: self-report and impaired scapular movements. Phys Ther 2006;86:1065-74.

6. Karduna AR, McClure PW, Michener LA, et al. Dynamic measurements of three-dimensional scapular kinematics: a validation study. J Biomech Eng 2001;123:184-90.

7. Lin JJ, Hanten WP, Olson SL, et al. Functional activity characteristics of individuals with shoulder dysfunctions. J Electromyogr Kinesiol 2005;15: $576-86$.

8. McQuade KJ, Dawson J, Smidt GL. Scapulothoracic muscle fatigue associated with alterations in scapulohumeral rhythm kinematics during maximum resistive shoulder elevation. J Orthop Sports Phys Ther 1998;28: $74-80$.

9. Matias R, Pascoal AG. The unstable shoulder in arm elevation: a threedimensional and electromyographic study in subjects with glenohumeral instability. Clin Biomech 2006;21:S52-8.

10. Paine RM, Voight M. The role of the scapula. J Orthop Sports Phys Ther 1993;18:386-91.
11. Borstad JD. Resting position variables at the shoulder: evidence to support a posture-impairment association. Phys Ther 2006;86:549-57.

12. Cools AM, Witvrouw EE, Declercq GA, et al. Scapular muscle recruitment patterns: trapezius muscle latency with and without impingement symptoms. Am J Sports Med 2003;31:542-9.

13. Horsley I. Assessment of shoulder with pain of a non-traumatic origin. Phys Ther Sport 2005;6:6-14.

14. Perotto AO. Anatomical Guide for the Electromyographer-The Limbs and Trunk. 3rd ed. Springfield: Charles C Thomas; 1994.

15. Hislop H, Montgomery J. Daniels and Worthingham's. Muscle Testing: Techniques of Manual Examination. 6th ed. Philadelphia: WB Saunders Company; 1995.

16. Lin JJ, Hanten WP, Olson SL, et al. Functional activities characteristics of shoulder complex movements: exploration with a three-dimensional electromagnetic measurement system. J Rehabil Res Dev 2005;42:199-210.

17. Day JS, Murdoch DJ, Dumas GA. Calibration of position and angular data from a magnetic tracking device. J Biomech 2000;33:1039-45.

18. Lee DC, Lim HK, McKay WB, et al. Toward an objective interpretation of surface EMG patterns: a voluntary response index (VRI). J Electromyogr Kinesiol 2004;14:379-88.

19. Lim HK, Lee DC, McKay WB, et al. Analysis of sEMG during voluntary movement. Part II: Voluntary response index sensitivity. IEEE Trans Neural Syst Rehabil Eng 2004;12:416-21.

20. Lin JJ, Lim HK, Soto-quijano DA, et al. Altered patterns of muscle activation during performance of four functional tasks in patients with shoulder disorders: interpretation from voluntary response index. J Electromyogr Kinesiol 2006;16:458-68.

21. Wu G, van der Helm FC, Veeger HE, et al. ISB recommendation on definitions of joint coordinate systems of various joints for the reporting of human joint motion. Part II: Shoulder, elbow, wrist and hand. J Biomech 2005;38: 981-92.

22. Portney LG, Watkins MP. Foundations of Clinical Research: Applications to Practice. 2nd ed. Conn: Appleton \& Lange, East Norwalk; 2000.

23. Theodoridis D, Ruston S. The effect of shoulder movements on thoracic spine 3D motion, Clin Biomech 2002;17:418-21.

24. Crosbie J, Kilbreath SL, Hollmann L, et al. Scapulohumeral rhythm and associated spinal motion. Clin Biomech 2008;23:184-92.

25. Edmonston SJ, Singer KP. Thoracic spine: anatomical and biomechanical considerations for manual therapy. Man Ther 1997;2:132-43.

26. Ascani E, Bartolozzi P, Logroscino C, et al. Natural history of untreated IS after skeletal maturity. Spine 1986;11:784-9.

27. Lawellin LD, Smith D, O'Brien MF, et al. Platelet calmodulin levels in adolescent idiopathic scoliosis: do the levels correlate with curve progression and severity? Spine 2002;27:768-75. 